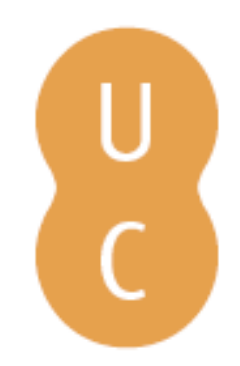

\title{
nommalina
}

\section{O papel da vulnerabilidade sísmica na mitigação: do risco sísmico de núcleos urbanos antigos}
Autor(es):
Vicente, Romeu; Ferreira, Tiago M.; Maio, Rui
Publicado por: $\begin{aligned} & \text { Imprensa da Universidade de Coimbra; RISCOS - Associação } \\ & \text { Portuguesa de Riscos, Prevenção e Segurança }\end{aligned}$
URL persistente:
URI:http://hdl.handle.net/10316.2/34824
DOI:
DOI:http://dx.doi.org/10.14195/978-989-96253-3-4_33
Accessed : $\quad$ 26-Apr-2023 10:36:46

A navegação consulta e descarregamento dos títulos inseridos nas Bibliotecas Digitais UC Digitalis, UC Pombalina e UC Impactum, pressupõem a aceitação plena e sem reservas dos Termos e Condições de Uso destas Bibliotecas Digitais, disponíveis em https://digitalis.uc.pt/pt-pt/termos.

Conforme exposto nos referidos Termos e Condições de Uso, o descarregamento de títulos de acesso restrito requer uma licença válida de autorização devendo o utilizador aceder ao(s) documento(s) a partir de um endereço de IP da instituição detentora da supramencionada licença.

Ao utilizador é apenas permitido o descarregamento para uso pessoal, pelo que o emprego do(s) título(s) descarregado(s) para outro fim, designadamente comercial, carece de autorização do respetivo autor ou editor da obra.

Na medida em que todas as obras da UC Digitalis se encontram protegidas pelo Código do Direito de Autor e Direitos Conexos e demais legislação aplicável, toda a cópia, parcial ou total, deste documento, nos casos em que é legalmente admitida, deverá conter ou fazer-se acompanhar por este aviso. 

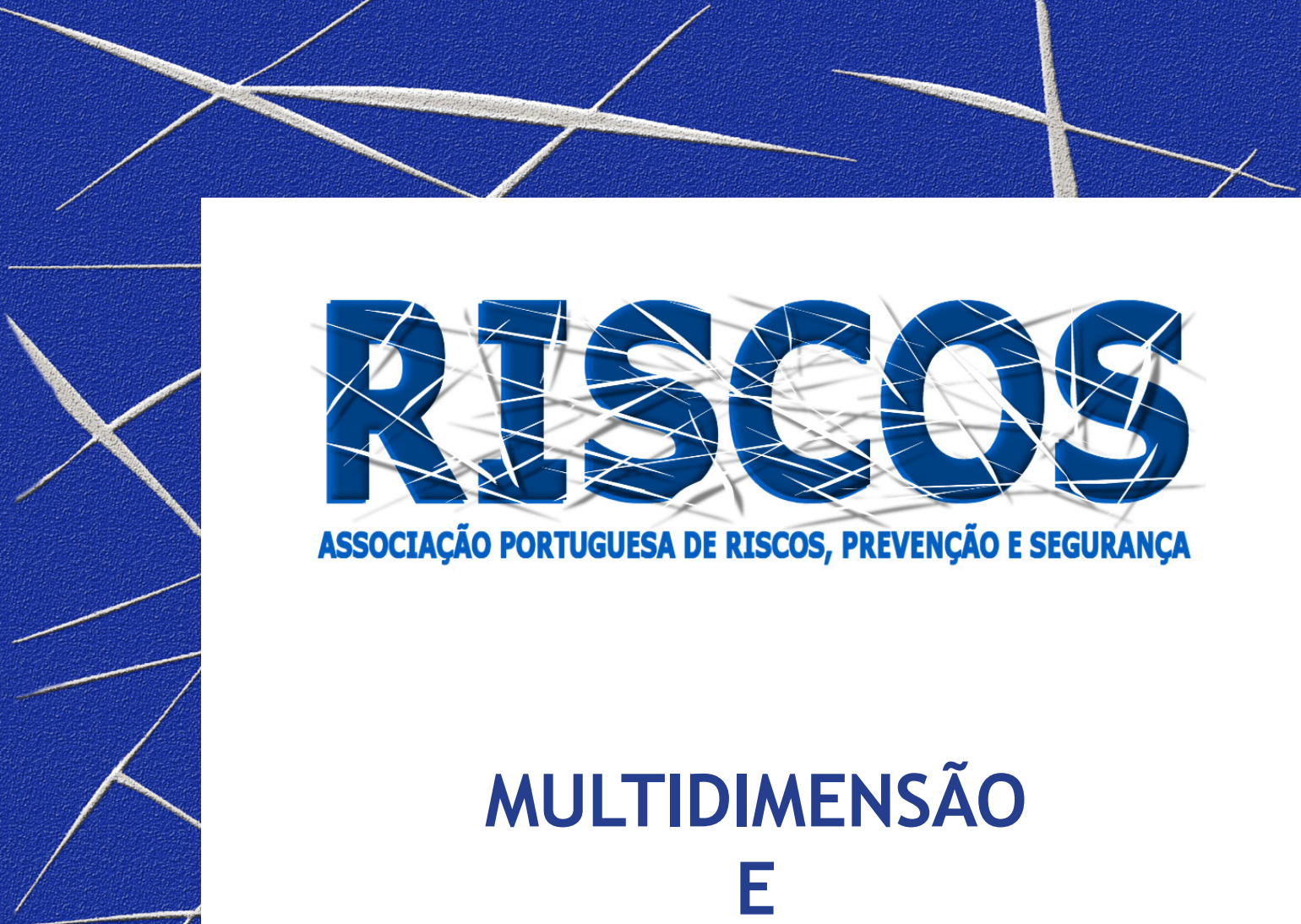

ASSOCIAÇÃO PORTUGUESA DE RISCOS, PREVENCCÃO E SEGURANÇA

MULTIDIMENSÃO

E
TERRITÓRIOS DE RISCO

III Congresso Internacional

I Simpósio Ibero-Americano

VIII Encontro Nacional de Riscos

Guimarães

2014 


\title{
O PAPEL DA VULNERABILIDADE SÍSMICA NA MITIGAÇÃO DO RISCO SÍSMICO DE NÚCLEOS URBANOS ANTIGOS
}

\author{
Romeu Vicente \\ Departamento de Engenharia Civil, Universidade de Aveiro \\ romvic@ua.pt \\ Tiago M. Ferreira \\ Departamento de Engenharia Civil, Universidade de Aveiro \\ tmferreira@ua.pt \\ Rui Maio \\ Departamento de Engenharia Civil, Universidade de Aveiro \\ ruiamaio@ua.pt
}

\begin{abstract}
RESUMO
Apesar da exposição a um determinado risco compreender responsabilidades políticas, económicas e sociais, na grande maioria dos casos, as estratégias de sensibilização e mitigação do risco sísmico surgem apenas na sequência de um evento de impacto significativo nas sociedades. Assim, a mitigação do risco é aqui abordada, incidindo na avaliação da vulnerabilidade sísmica de núcleos urbanos antigos, zonas particularmente vulneráveis das nossas cidades, na tentativa de contrariar esta tendência. Com esse objectivo aplicou-se uma metodologia simplificada que possibilitou a estimativa de cenários de dano e de perdas económicas e humanas. Através do mapeamento destes resultados, integrados num Sistema de Informação Geográfica, poderão ser elaborados planos estratégicos de reabilitação do edificado urbano, de emergência e evacuação em caso de ocorrência sísmica.

Palavras-chave: risco sísmico; vulnerabilidade sísmica; índice de vulnerabilidade; núcleos urbanos antigos; cenários de dano; estimativa de perdas.
\end{abstract}

Introdução

Este artigo aborda a redução da vulnerabilidade do edificado como a medida mais sustentável na mitigação do risco sísmico. Dada a incerteza associada à ocorrência de um evento, a perigosidade sísmica é, de entre os três vetores definidores do risco, aquele que não é passível de ser minimizado. Quanto à exposição, a qual avalia o valor dos elementos em risco (humanos e materiais), esta pode contribuir para a mitigação do risco sísmico através da adopção de medidas no sentido de condicionar a construção e o desenvolvimento das cidades em locais de elevada perigosidade sísmica. Desta forma, a redução da vulnerabilidade sísmica do edificado apresenta-se como a estratégia mais sustentável na mitigação do risco sísmico.

\section{O centro histórico de Faro como caso de estudo}

O Bairro Ribeirinho do núcleo urbano antigo de Faro, Portugal, foi a zona de estudo escolhida para a avaliação da vulnerabilidade sísmica segundo esta metodologia. A sua seleção baseou-se no facto de esta ser uma das zonas com elevada concentração de edifícios em alvenaria, muitos destes com níveis de alteração estrutural muito reduzidos. Refira-se ainda que, de forma a melhorar e optimizar a gestão de todas as propriedades do parque edificado, foi desenvolvido e utilizado um sistema de informação em software ArcGis $10.2^{\circledR}$ (ESRI, 2005), o qual combina dados georreferenciados com informação relativa a cada edifício avaliado.

\section{Metodologia do Índice de Vulnerabilidade}

A formulação do índice de vulnerabilidade foi originalmente desenvolvida em Itália pelo GNDT II (1994), para a avaliação da vulnerabilidade sísmica de edifícios de alvenaria através da observação 
e catalogação de danos e mecanismos após a ocorrência de um evento sísmico. Em Portugal, esta metodologia foi adaptada à realidade dos nossos edifícios por Vicente (2011), introduzindo uma análise e inspeção mais cuidada e ainda outros novos parâmetros contabilizando a interação entre edifícios adjacentes. Inicialmente aplicada à Baixa da cidade de Coimbra (Vicente, 2011), esta metodologia foi recentemente utilizada na avaliação do núcleo urbano antigo do Seixal (Ferreira et al., 2013). A metodologia assenta na determinação de um índice de vulnerabilidade, $I_{v}$, o qual é calculado para cada edifício através de uma média ponderada de 14 parâmetros distribuídos em 4 classes de vulnerabilidade crescente, $C_{v i}$, de $A$ a $D$, possuindo diferentes pesos relativos, $p_{i}$, consoante a sua relevância no comportamento sísmico do edifício. A definição detalhada de cada um destes 14 parâmetros pode ser consultada em Vicente (2011).

\section{Resultados}

A aplicação da metodologia do índice de vulnerabilidade a cada um dos 191 edifícios avaliados é apresentada na Figura 1 (a), através do mapeamento do $I v$ para cada edifício. Na Figura 1 (b) são identificados os edifícios com um valor de $I v$ superior a 40 , e que deverão por isso, ser motivo de maior atenção no estabelecimento de prioridades e estratégias de intervenção. Note-se que aproximadamente $15 \%$ dos edifícios apresentam um Iv superior a 40 , com $5 \%$ acima de 45 (equivalente a uma classe de vulnerabilidade A na escala EMS-98). Por outro lado, apenas $4 \%$ dos edifícios avaliados apresentam um $I V$ inferiores a 20 (equivalente a uma classe de vulnerabilidade B na escala EMS-98).

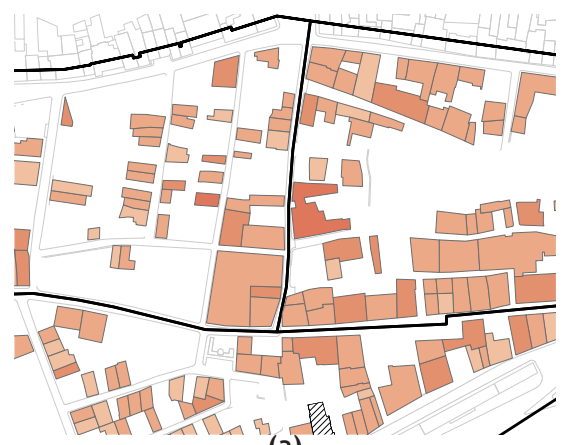

(a)

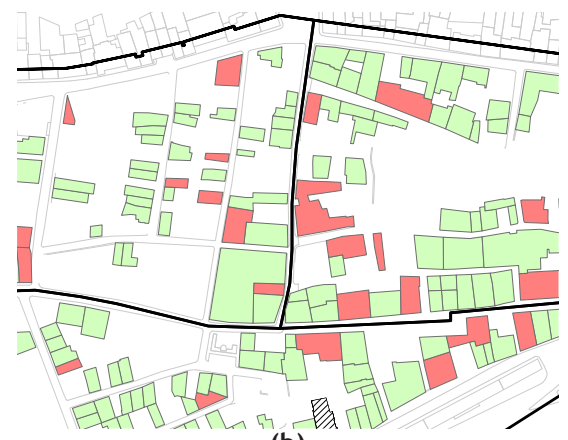

(b)

Figura 1. Mapeamento do índice de vulnerabilidade para o Bairro Ribeirinho de Faro (a) e identificação dos edifícios com $I_{v}>40$ (b).

Relativamente aos valores médios do índice de vulnerabilidade, $I_{v, \text { mean }}$, foi efetuada uma primeira avaliação considerando apenas os 53 edifícios avaliados detalhadamente para a qual foi obtido um $I_{v \text {, mean }}$ igual a 36.15 .

De acordo com esta metodologia, o grau médio de dano, $\mu_{D}$, pode ser estimado para diferentes intensidades através da aplicação da Eq. (1), desenvolvida para esse fim por Bernardini et al. (2007). 


$$
\mu_{D}=2.5 \times\left[1+\tanh \left(\frac{I+6.25 \times V-13.1}{Q}\right)\right] ; 0 \leq \mu_{D} \leq 5
$$

onde I representa a perigosidade sísmica descrita em termos de intensidade macrossísmica, $V$ representa o índice de vulnerabilidade (ver Eq. (2)), e onde $Q$ é o factor de ductilidade que descreve a ductilidade de certas tipologias construtivas (considerado igual a 3.0). A Figura 2 (a) e (b) apresenta os cenários de grau médio de dano para as intensidade macrossísmicas, I (EMS-98), $_{\text {, }}$, VIII e IX, respetivamente.

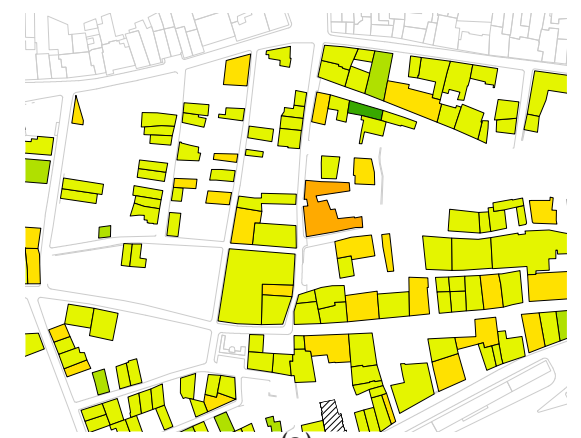

(a)

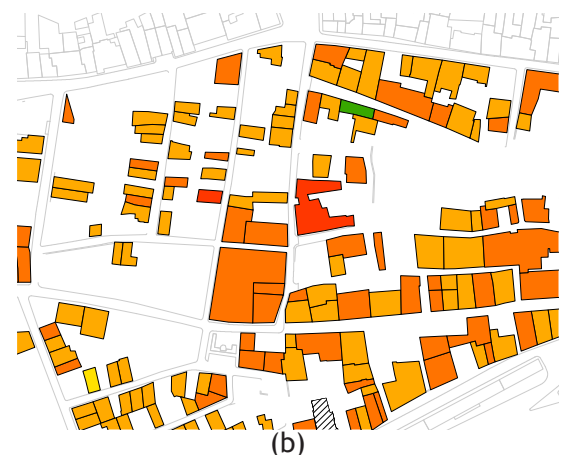

(b)

Figura 2. Cenários de dano para intensidades $I_{(\mathrm{EMS}-98)}=$ VIII (a) e $I_{(\mathrm{EMS}-98)}=\mathrm{IX}(\mathrm{b})$.

A estimativa de edifícios colapsados e inutilizáveis, de extrema utilidade para as entidades governamentais e de proteção civil, foi neste artigo avaliada com base num modelo desenvolvido por Bramerini et al. (1995) que define os estados de dano que relacionam a probabilidade de se exceder um determinado grau de dano com a probabilidade de colapso e perda funcional. A estimativa do número total de edifícios colapsados e inutilizáveis para diferentes intensidades sísmicas, $I_{(E M S-98)}$, e para o valor $I_{v, \text { mean }}=34.12$, encontra-se apresentada na Figura 3.

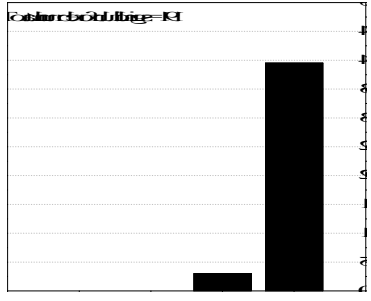

(a)

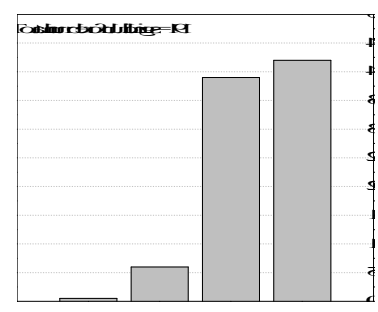

(b)

Figura 3. Estimativa do número de edifícios colapsados (a) e inutilizáveis (b). 
Também na avaliação das perdas humanas e número de desalojados foi utilizado o modelo desenvolvido por Bramerini et al. (1995). Para isso, definiu-se a percentagem de mortos e feridos graves como sendo $30 \%$ do número total de habitantes residentes em edifícios colapsados e inutilizáveis. A estimativa do número de mortos e feridos graves e de desalojados, para o $I_{v, \text { mean }}$ = 34.12, é apresentada na Figura 4. Note-se que estes números podem ser significativamente agravados em função da sazonalidade própria da região do Algarve e da hora a que ocorra o sismo.

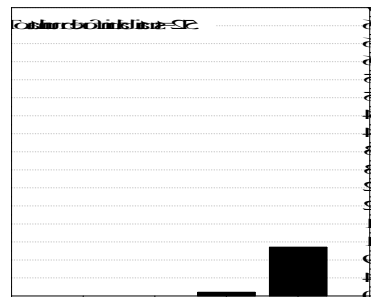

(a)

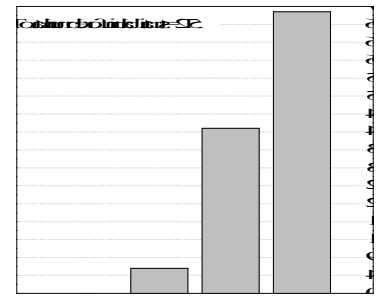

(b)

Figura 4. Estimativa do número de mortos e feridos graves (a) e desalojados (b).

De acordo com Vicente et al. (2011) a probabilidade de custo de reparação para um determinado evento sísmico caracterizado por uma intensidade $I, P[R \mid I]$, pode ser calculada através do produto da probabilidade condicional do custo de reparação para cada grau de dano, $P\left[R \mid D_{k}\right]$ com a probabilidade condicional de condição de dano para cada nível de vulnerabilidade e intensidade sísmica, $P\left[D_{k}\left|I_{v}\right|\right]$. Assim, de forma a estimar o custo de reparação associado a diferentes valores de vulnerabilidade na estimativa de perdas, considerou-se um custo unitário de $482 € / \mathrm{m}^{2}$ em função do valor sugerido pela Portaria n³70/2013 (Portugal, 2013). A Figura 5 apresenta a estimativa global dos custos de reparação para o Bairro Ribeirinho de Faro.

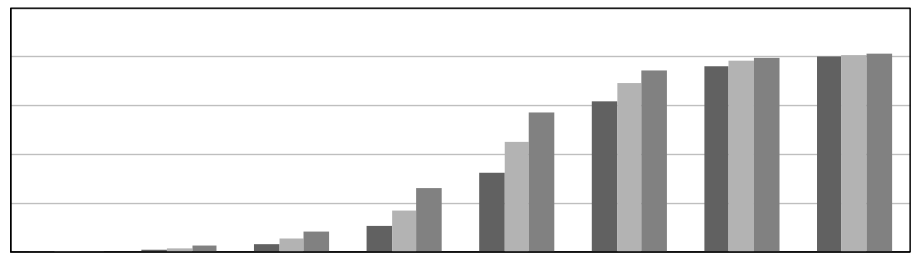

Figura 5. Estimativa dos custos de reparação.

\section{Comentários Finais}

De um modo geral os resultados estão coerentes com as características e o estado de conservação do edificado do Bairro Ribeirinho de Faro. Estando associada à cidade de Faro uma perigosidade sísmica moderada, estes resultados revelam que a ocorrência de evento sísmico de intensidade 
moderada poderá causar danos e perdas significativas, motivadas, quer pela vulnerabilidade do próprio edificado, quer pela falta de preparação das entidades responsáveis e da população. Por último, sublinhe-se que uma avaliação rigorosa e competente da vulnerabilidade sísmica dos núcleos históricos antigos, complementada com soluções apropriadas de reforço estrutural e sísmico, podem reduzir significativamente os danos e as perdas humanas e económicas causados por futuros eventos.

\section{Agradecimentos}

Este trabalho foi financiado pela Fundação para a Ciência e a Tecnologia (FCT) ao abrigo do projeto de investigação URBSIS: Avaliação da Vulnerabilidade e Gestão do Risco Sísmico à Escala Urbana (PTDC/ECM-URB/2564/2012).

\section{Bibliografia}

Bernardini, A.; Giovinazzi, S.; Lagomarsino, S. e Parodi, S. (2007) - Vulnerabilità e previsione di danno a scala territoriale secondo una metodologia macrosismica coerente con la scala EMS-98. ANIDIS, In: XII Convegno Nazionale l'ingegneria sismica in Italia. 10-14 Junho, Pisa, Itália

Bramerini, F.; Di Pasquale, G.; Orsini, A.; Pugliese, A.; Romeo, R. e Sabetta, F. (1995) - Rischio sismico del territorio italiano: proposta per una metodologia e risultati preliminari. Servizio Sismico Nazionale, Relatório Técnico SSN/RT/95/01, Roma;

ESRI (2005) - Geographic Information Systems. Nova lorque n³10, Redlands, 92373-8100, E.U.A.

Ferreira, T.M.; Vicente, R.; Mendes da Silva, J.A.R.; Varum, H. e Costa, A. (2013) - Seismic vulnerability assessment of historical urban centres: case study of the old city centre in Seixal, Portugal. Bulletin of Earthquake Engineering, Springer, vol. 11, p. [1753-1773], DOI: 10.1007/s10518-013-9447-2;

GNDT (1994) - Scheda di esposizione e vulnerabilità e di rilevamento danni di primo e secondo livello (murata e cemento armato), Gruppo Nazionale per la Difesa dai Terremoti, Roma, Itália.

Portugal (2013) - Portaria n³70/2013, de 27 de Dezembro de 2013. Ministério do Ambiente e do Ordenamento do Território, Diário da República, $1^{\text {a }}$ série, $n^{\circ} 251$, Portugal.

Vicente, R.S.; Parodi, S.; Lagomarsino, S.; Varum, H. (2011) - Seismic vulnerability and risk assessment: case study of the historic city centre of Coimbra, Portugal. Bulletin of Earthquake Engineering, Springer, Holanda, vol. 9(4), p. [1067-1096]; 